\title{
Targeting disease by immunomodulation
}

\author{
T Kaufmann ${ }^{1}$ and HU Simon ${ }^{*, 1}$ \\ Cell Death and Differentiation (2015) 22, 185-186; doi:10.1038/cdd.2014.166
}

Immunology is a highly complex field and, upon screening of the primary research literature, newcomers to the field are often overwhelmed by the seemingly incomprehensible number of publications, becoming lost in highly specialized subfields and terminology without grasping the 'big picture'. Of course, one would first consult textbooks; however, with the former lagging several years behind the cutting edge, it is primarily review articles on which scientists rely to sieve through the primary literature, to grasp the important bits and pieces, and to connect seemingly unrelated findings. Thus, every good review article is an invaluable aid, bringing new thinkers up to speed and adding to the diversity of ideas.

There is no doubt that all the different arms of the immune system are strongly interconnected and that the different cross-talk connections and regulatory mechanisms that are in place need to be finely tuned to maintain the health of the organism. Defects in the regulation of the immune system are implicated in the pathogenesis of chronic inflammatory diseases and cancer. In this issue of Cell Death and Differentiation, a set of five reviews highlights the interplay of innate and adaptive immunity in health and disease. The authors of these articles discuss the links (and underlying mechanisms) between deregulated immune cells and autoimmune disorders or cancer, and present the current and putative novel strategies to target these cells with immunomodulatory compounds for a beneficial therapeutic outcome.

The innate immune system serves to mount an inflammatory response as a first line of defense against invading pathogens and then initiates the subsequent immune responses. Over the recent years, innate immune signaling has persisted as a 'hot topic' in immunology and important advances in understanding the underlying biology have been achieved. Humphries et al. ${ }^{1}$ introduce us to the family of receptor interacting protein kinases (RIP), in particular RIP1, 2 , and 3 , and their role in inflammatory signaling, apoptosis, and inflammation-associated cell death. RIP1 mediates proinflammatory cytokine expression downstream of 'death ligands' (e.g. tumor necrosis factor alpha) and, upon encountering 'danger signals' (e.g. bacterial lipopolysaccharide or viral RNA), it is involved in the switch to apoptosis signaling for the elimination of pathogens replicating within host cells. Interestingly, some microbes have evolved to actively block apoptosis of the host cell, which in turn can activate a RIP1/ RIP3-dependent inflammatory cell death, called necroptosis, in order to prevent further spreading of the pathogen. ${ }^{2}$ RIP3, which interestingly also seems to control the activity of the inflammasome, ${ }^{3,4}$ is increasingly recognized as having an important role in the pathogenesis of several inflammatory diseases. On the other hand, RIP2 has emerged as a critical component in NOD1/2 signaling, which is activated specifically in response to intracellular bacteria. Better understanding of these pathways seems to be of high clinical relevance. For instance, loss of function mutations in NOD2 predispose to the development of Crohn's disease.

Dendritic cells (DC) orchestrate the transition from the early innate immune response to the activation of adaptive immunity. DCs are central for the establishment of central and peripheral tolerance. ${ }^{5-7}$ With a focus on multiple sclerosis (MS), Quintana et al. ${ }^{8}$ discussed how abnormalities in DC function contribute to the pathology of MS and how the therapeutic use of immunomodulatory drugs modulates DC function. The anti-inflammatory role of the cytokine interleukin27 (IL-27) in DCs is emphasized with regard to therapeutic applicability. The authors present aryl hydrocarbon receptor, a likely mediator downstream of IL-27, as a promising target with the advantage of fewer side effects than direct IL-27 administration. Nanoparticle-mediated codelivery of ligands activating tolerogenic DC signaling together with myelinspecific antigens is introduced as a potential novel strategy to modulate the function of DCs in vivo in MS patients.

$\mathrm{IL}-12$, originally described as a natural killer cell-stimulatory factor, ${ }^{9}$ is an early proinflammatory, Th1-polarizing, cytokine that links the adaptive and innate immune system. IL-12 has emerged as a potent inducer of antitumor immunity in preclinical trials, whereas its acts on multiple immune cells that form the tumor microenvironment. However, as pointed out by Tugues et al., ${ }^{10}$ translation into the clinics has not been straightforward, partly due to the lack of antitumor response, but also due to toxic side effects of IL-12. Promising novel strategies that limit unwanted side effects focus on targeted delivery of IL-12 using gene therapy, nanoparticles, immunecytokines or genetically engineered IL-12-producing cells to the tumor site. Even though IL-12-based immunotherapy still awaits FDA approval, the authors speculate that such approaches will likely be most successful as only a part of combination therapies, with the aim to increase the immunogenicity of tumor cells.

It is well accepted that the gut microbiota formed by commensals is indispensable for health because of its many functions, including protection from pathogen infection,

\footnotetext{
${ }^{1}$ Institute of Pharmacology, University of Bern, Bern, Switzerland

*Corresponding author: HU Simon, Institute of Pharmacology, University of Bern, Friedbuehlstrasse 49, CH-3010 Bern, Switzerland. Tel: +4131 63232 81; Fax: +4131 632 49 92; E-mail: hus@pki.unibe.ch
} 
instruction of the developing immune system and critical support of digestion. Earlier dogma was that chemo- or radiotherapy-induced damage of the intestinal epithelium negatively influenced the outcome of tumor therapy. There is, however, increasing evidence that cyclophosphamide or platinum salt-based anticancer treatment is less efficient in germ-free mice or mice treated with antibiotics. Viaud et al. ${ }^{11}$ highlight these beneficial effects of gut commensals ('really hot sh*t', as it was put by the authors) in cancer therapy. Underlying mechanisms include the reprogramming of intratumoral myeloid cells by gut microbiota and translocation of specific Gram-positive bacteria to secondary lymphoid organs to prime specialized T cells. It is proposed that in the future analysis of the patient's microbiota should be an integral part of personalized therapy. Restoring an optimal microbial composition, rather than eradicating the whole gut microflora, may increase the efficacy of chemotherapeutics.

Riether et al. ${ }^{12}$ lastly provide a comprehensive insight into leukemic stem cells (LSC) and a comparison to hematopoietic stem cells (HSC), with which they share many features. The cancer stem cell hypothesis was first proposed in leukemia and also applies to several solid tumors. It postulates that within a leukemic tumor a small population with stem cell-like characteristics, the leukemic stem cell, is responsible for initiating and maintaining the disease and that more differentiated cancer cells were produced by asymmetric division. ${ }^{13,14}$ This review article sheds light on similarities and differences between LSCs and HSCs, their respective niches within the bone marrow (BM) and the regulation of the BM microenvironment by cells of the adaptive and innate immune system. Paradoxically, mechanisms that have evolved in the BM to maximize the response towards acute infection seem to contribute to leukemia progression. The authors underline the necessity for eliminating the highly resistant LSCs in order to cure leukemia and that an improved understanding of the so-called 'immune niche' with its complex interplay involving immune cells, nonimmune cells and secreted factors, may help to develop improved strategies for immunotherapies.

Taken together, the review series in this issue of Cell Death and Differentiation highlights the role of the immune system in the pathogenesis of multiple diseases, including cancer, with five excellent contributions. Therapeutic advances will not be possible without clarification of the major pathways leading to the immune dysregulation associated with these diseases. One hopes that more specific therapeutics in conjunction with novel diagnostic assays will be developed in the upcoming years, contributing to a new approach which is currently being undertaken in medicine called 'stratified' medicine.

\section{Conflict of Interest}

The authors declare no conflict of interest.

1. Humphries F et al. Cell Death Differ 2014; doi:10.1036/cdd.2014.126.

2. Khan $\mathrm{N}$ et al. Curr Opin Immunol 2014; 26: 76-89.

3. Vince JE et al. Immunity 2012; 36: 215-227.

4. Yabal $\mathrm{M}$ et al. Cell Rep 2014; $7: 1796-1808$.

5. Hawiger D et al. J Exp Med 2001; 194: 769-779.

6. Peterson P, Org T, Rebane A. Nat Rev Immunol 2008; 8: 948-957.

7. Steinman RM. Ann Rev Immunol 2012; 30: 1-22.

8. Quintana FJ, Yeste A, Mascanfroni ID. Cell Death Differ 2014; doi:10.1036/cdd.2014.125.

9. Kobayashi $\mathrm{M}$ et al. J Exp Med 1989; 170: 827-845.

10. Tugues $\mathrm{S}$ et al. Cell Death Differ 2014; doi:10.1036/cdd.2014.134

11. Viaud S et al. Cell Death Differ 2014; doi:10.1036/cdd.2014.56.

12. Riether C, Schurch CM, Ochsenbein AF. Cell Death Differ 2014; doi:10.1036/cdd.2014.89.

13. Lapidot T et al. Nature 1994; 367: 645-648.

14. Reya T, Morrison SJ, Clarke MF, Weissman IL. Nature 2001; 414: 105-111. 